\title{
Fabrication of autocloned photonic crystals using electron-beam guns with ion-assisted deposition
}

\author{
Te-Hung Chang ${ }^{\mathrm{a}, *}$, Sheng-Hui Chen ${ }^{\mathrm{a}, 1}$, Cheng-Chung Lee ${ }^{\mathrm{a}, 1}$, Husen-Li Chen ${ }^{\mathrm{b}}$ \\ ${ }^{\mathrm{a}}$ Department of Optics and Photonics and Thin Film Technology Center National Central University, 320, Taiwan \\ ${ }^{\mathrm{b}}$ Department of Material Science and Engineering, National Taiwan University, Taipei, Taiwan
}

Received 6 September 2006; received in revised form 28 May 2007; accepted 31 May 2007

Available online 13 June 2007

\begin{abstract}
The autocloning technique is an attractive deposition method for the making of photonic crystals. With it various photonic crystals can be produced simply by changing the substrate periodicity and the structure of the stacking materials. We report on a method for the fabrication of autocloned photonic crystals. This method has better step-coverage, a higher deposition rate and a larger deposition area than can be achieved by the more traditional sputtering method and the periodic surface corrugation is preserved even after ion-assisted deposition (IAD) of multilayer stacks using E-beam gun evaporation. The shaping process can be freely controlled by controlling the IAD power and the ion source etching time. Ion source etching is a physical etching process which does not require any chemical reaction or dangerous reactive gas. The process parameters are described in this paper. The refractive index can be adjusted by changing the deposition rate and the substrate temperature during the deposition process. The deposition rate is about $0.7-1 \mathrm{~nm} / \mathrm{s}$ for $\mathrm{SiO}_{2}$ which is almost ten times faster than that of the sputtering method. This makes this method good for the mass production of photonic crystals.

(C) 2007 Elsevier B.V. All rights reserved.
\end{abstract}

Keywords: Thin-film; Autocloning; Photonic crystal; Ion-assisted deposition; Ion etching; Scanning electron microscopy

\section{Introduction}

Since first being discussed theoretically in 1987 by E. Yablonovitch [1] and S. John [2], photonic crystals (PhCs) have continued to gain much interest in both fundamental and applied research. PhCs are nanostructures that consist of two or more periodically ordered materials with different refractive indices. The refractive index in a $\mathrm{PhC}$ varies periodically on a scale on the order of the wavelength of light. The propagation of electromagnetic waves of certain frequency ranges is either allowed or forbidden in the same manner as the periodic potential for electrons in atomic crystals. In nature, examples of

\footnotetext{
* Corresponding author. Tel.: +886 34263918.

E-mail addresses: thchang@dop.ncu.edu.tw (T.-H. Chang), cclee@dop.ncu.edu.tw (C.-C. Lee).

URL's: http://www.ncu.edu.tw/ tftc (T.-H. Chang), http://www.ncu.edu.tw/ tftc (S.-H. Chen), http://www.ncu.edu.tw/ tftc (C.-C. Lee)

${ }^{1}$ Tel.: +886 34263918 .
}

the optical properties of PhCs can be seen in opals, the wings of the Morpho butterfly [3], the spines of the sea mouse [4], and some other animals $[5,6]$. The optical properties behave as gleaming colors, even in the absence of pigments. This is because the periodic structures of the PhCs allow strong wavelength sensitivity, controlling the propagation of light.

The theoretical basis for how PhCs function has often been studied over the last decade; however, three-dimensional (3D)$\mathrm{PhC}$ components or devices have not yet been developed commercially, due to the difficulty of fabricating periodic nanostructures. One attractive fabrication method that has been developed is called the "autocloning technology" and has shown its feasibility for 3D PhCs [7]. The process begins with the lithographic patterning of a periodic corrugation pattern on a substrate. Two kinds of dielectric films are then stacked alternately on the substrate by thin-film deposition and sputter etching to form a zigzag multilayer coating. This technology has been widely applied to the fabrication of 3D-PhC components in devices such as polarizers, waveguides, bandpass filters, and so on. In recent years sputtering [8] and plasma enhanced 


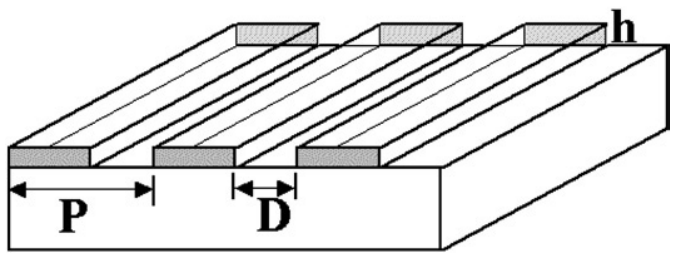

Fig. 1. Structure of the periodic striped corrugation patterns on the substrate

chemical vapor deposition [9] methods have been applied to the fabrication of 3D PhCs with autocloning technology.

In this study 3D PhCs have been fabricated by first patterning substrates with periodic corrugation patterns after which multilayer stacks have been fabricated by using E-beam gun evaporation ion-assisted deposition (IAD). The results show that the periodic surface corrugations are preserved very well after the deposition of multilayer structures given the appropriate IAD parameters. In addition, the slopes of the corrugations can be adjusted without changing the corrugation pattern of the substrate, simply by controlling the parameters of the ion beam.

\section{Experiments}

In this study, periodic striped corrugation patterns were patterned by electron-beam lithography (WEPRINT 200, LEICA) on silicon wafers. Fig. 1 illustrates the structure, where $P$ is the period, $D$ the width of the groove, and $h$ the depth of the groove. The IAD coating system used for the thinfilm process consisted of a $1100 \mathrm{~mm}$ box coater equipped with two E-beam guns operated at a voltage of $10 \mathrm{kV}$. A $500 \mathrm{~W}$ Kaufman-type Radio-frequency (RF) ion source with an RF neutralizer (RFN), made by Veeco Ion Tech Inc was used. The working gases of the RF ion source and RFN are oxygen $\left(\mathrm{O}_{2}\right)$ and argon (Ar), respectively. The deposition of the high index material, $\mathrm{Ta}_{2} \mathrm{O}_{5}$, was about $0.25 \mathrm{~nm} / \mathrm{s}$, and the deposition rate of the low index material, $\mathrm{SiO}_{2}$, was about $0.6 \mathrm{~nm} / \mathrm{s}$.

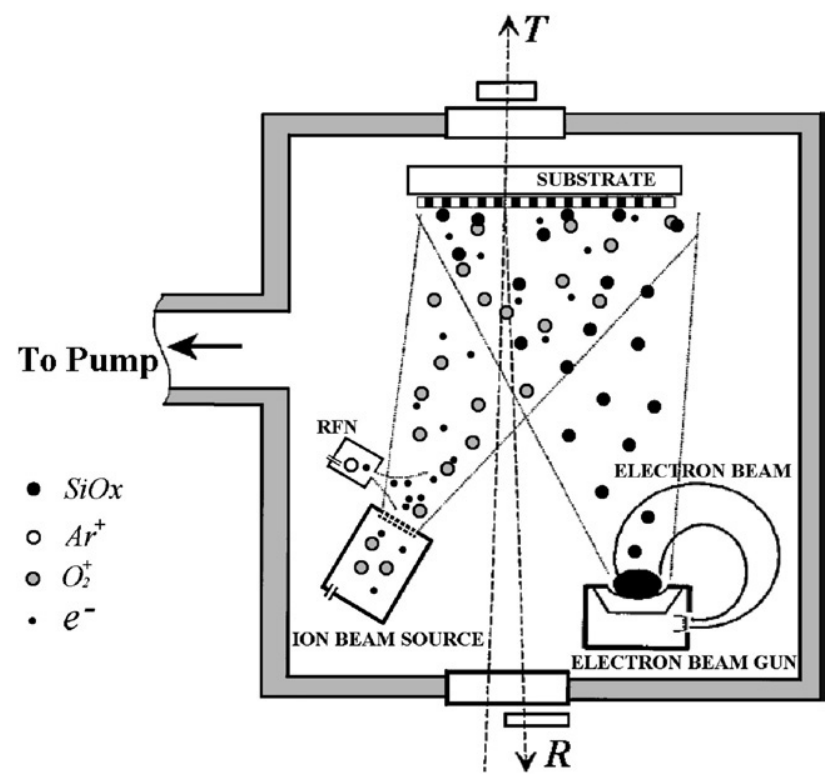

Fig. 2. Schematic drawing of the IAD coating system.
Table 1

E-beam gun and IAD Parameters

\begin{tabular}{lll}
\hline & Thin-film deposition & Ion etching \\
\hline Gas flow (sccm) ion source & $\mathrm{O}_{2}: 40$ & $\mathrm{O}_{2}: 40$ \\
Ion-beam voltage $(\mathrm{V})$ & 400 & $300-600$ \\
Ion-beam current $(\mathrm{mA})$ & 500 & 500 \\
Process pressure $(\mathrm{Pa})$ & 0.02666 & 0.02666 \\
Substrate temperature & Room temperature & Room temperature \\
\hline
\end{tabular}

The coating chamber was pumped to a base pressure of $1.066 \times 10^{-3} \mathrm{~Pa}$ before the thin-film process. The patterned substrates were placed $90 \mathrm{~cm}$ away from the E-beam guns. The
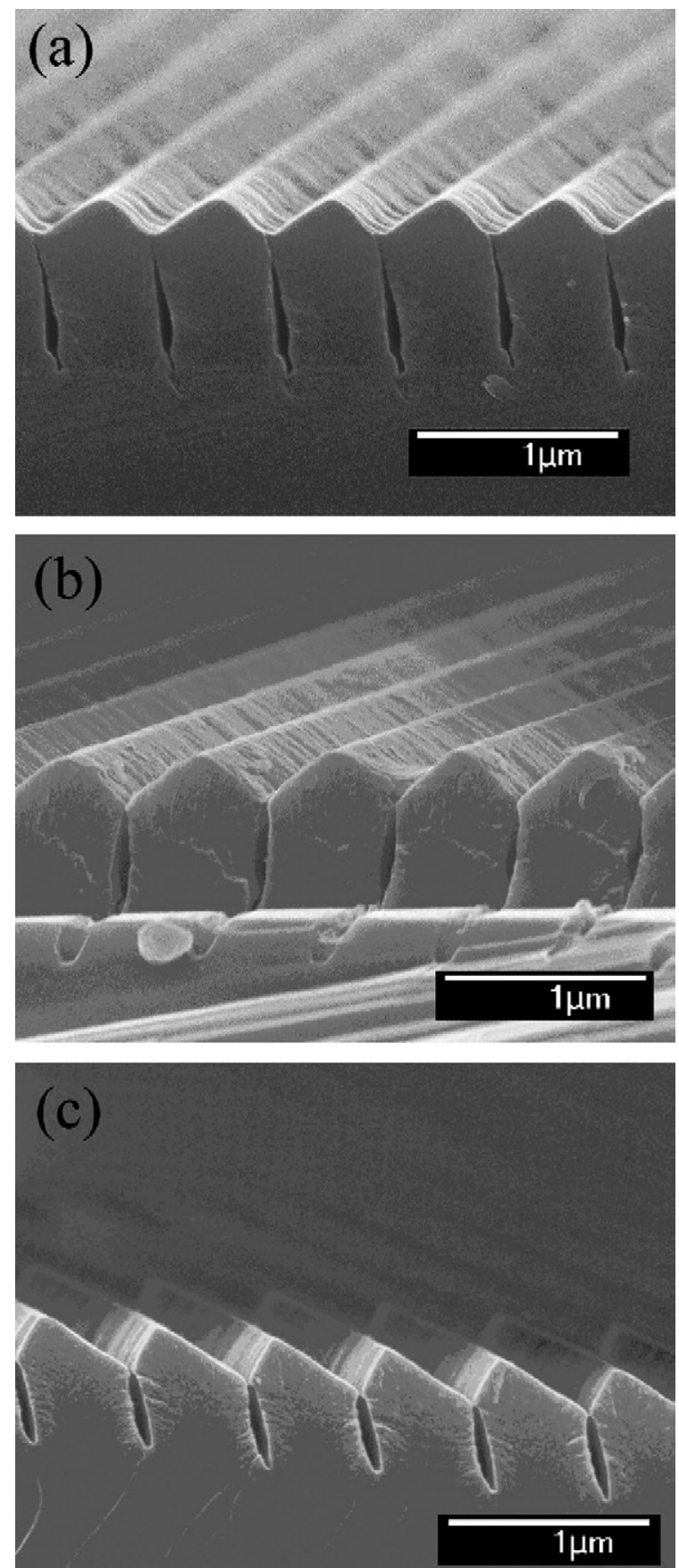

Fig. 3. IAD autocloning process with grooves of (a) $0.2 \mu \mathrm{m}$, (b) $0.3 \mu \mathrm{m}$, and (c) $0.4 \mu \mathrm{m}$ in depth. 
working pressure during the thin-film process was fixed at $2.666 \times 10^{-2} \mathrm{~Pa}$ by controlling the oxygen flow. Fig. 2 schematically depicts the coating system, while Table 1 describes the parameters of the experiments.

The autocloning process involves thin-film deposition, ionbeam etching, and redepositioning of the film particles. The surface shape assumes stationary states when films are formed under appropriate thin-film deposition, ion-beam etching and redepositioning conditions. Moreover, an important layer, called the "adjusting layer", has to be deposited between the patterned substrate and the multilayer stack. The shape of the surface of the adjusting layer had to reproduce the multilayers. The effects of the different parameters including the depths of the grooves, the ion-beam voltage and the ion-etching time of the autocloning process were analyzed. The material used for the adjusting layers was $\mathrm{SiO}_{2}$. The deposition thickness was about $0.8 \mu \mathrm{m}$. The period $P$ of the grooves was $0.6 \mu \mathrm{m}$ and the width of the grooves $D$ was $0.36 \mu \mathrm{m}$.

\subsection{Effect of the depth of the grooves}

The effect of the depth of the grooves was compared with the ion-beam source parameters: the ion-beam voltage was $400 \mathrm{~V}$ and the ion-etching time was $40 \mathrm{~min}$. The period of the grooves was $0.6 \mu \mathrm{m}$. Fig. 3(a), (b) and (c) show the self-shaping processes for grooves of $0.2 \mu \mathrm{m}, 0.3 \mu \mathrm{m}$ and $0.4 \mu \mathrm{m}$ in depth, respectively. The corrugation slopes of Fig. 3(a), (b) and (c) are $100^{\circ}, 103^{\circ}$ and $90^{\circ}$, respectively. The results show that the initial depth of the grooves had little effect on the corrugation slope. Furthermore even when the initial grooves were different in depth, the triangular shape of the zigzag structures of the adjusting layers remained similar.

\subsection{Effect of the ion-beam voltage}

The workable range of the ion-beam voltage of the Kaufmantype RF ion source from Veeco Ion Tech Inc is between $200 \mathrm{~V}$ and $1100 \mathrm{~V}$. The lower the ion-beam voltage, the more unstable the ion beam, and the higher the ion-beam voltage, the more absorption the film would have. In this experiment the ion-beam voltage was controlled between $300 \mathrm{~V}$ and $600 \mathrm{~V}$. Fig. 4(a), (b), (c) and (d) show self-shaping processes when the ion-beam voltages were $300 \mathrm{~V}, 400 \mathrm{~V}, 500 \mathrm{~V}$, and $600 \mathrm{~V}$, respectively.

In Fig. 4(a) it can be seen that at $300 \mathrm{~V}$, the ion-beam voltage was too low to provide enough energy for the ion beam to etch the structured film. Thus the cross-section of the adjusting layer has an arc structure rather than a zigzag structure. Fig. 4(b) shows that at $400 \mathrm{~V}$ the ion-beam had enough energy to etch the film. Thus the cross-section of the adjusting layer has a zigzag structure which was good for the autocloning process. However, when the ion-beam voltages were $500 \mathrm{~V}$ and $600 \mathrm{~V}$, the effect of ion etching exceeded the appropriate values. Fig. 4(c) and (d)
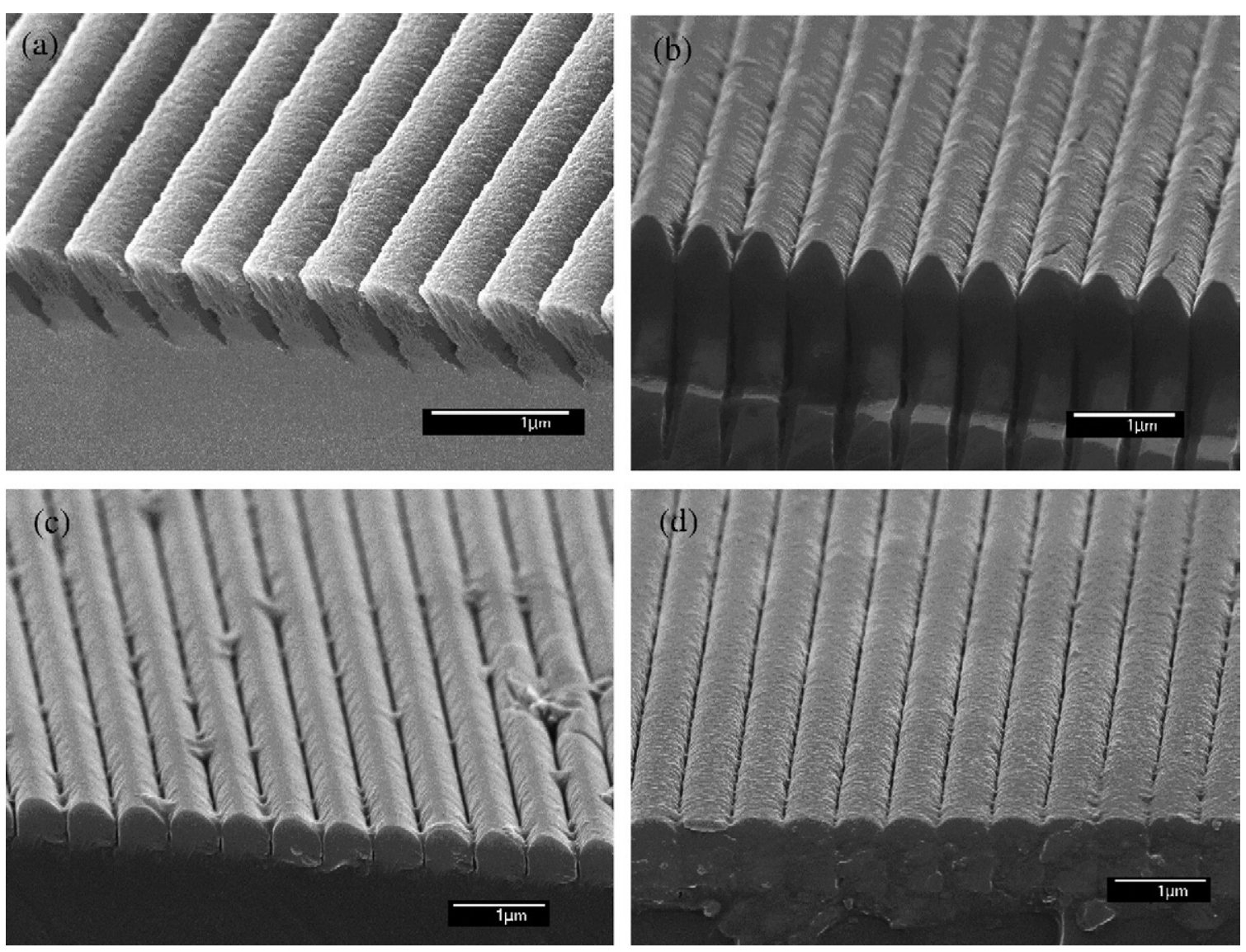

Fig. 4. IAD autocloning process with ion-beam voltages of (a) $300 \mathrm{~V}$, (b) $400 \mathrm{~V}$, (c) $500 \mathrm{~V}$ and (d) $600 \mathrm{~V}$. 

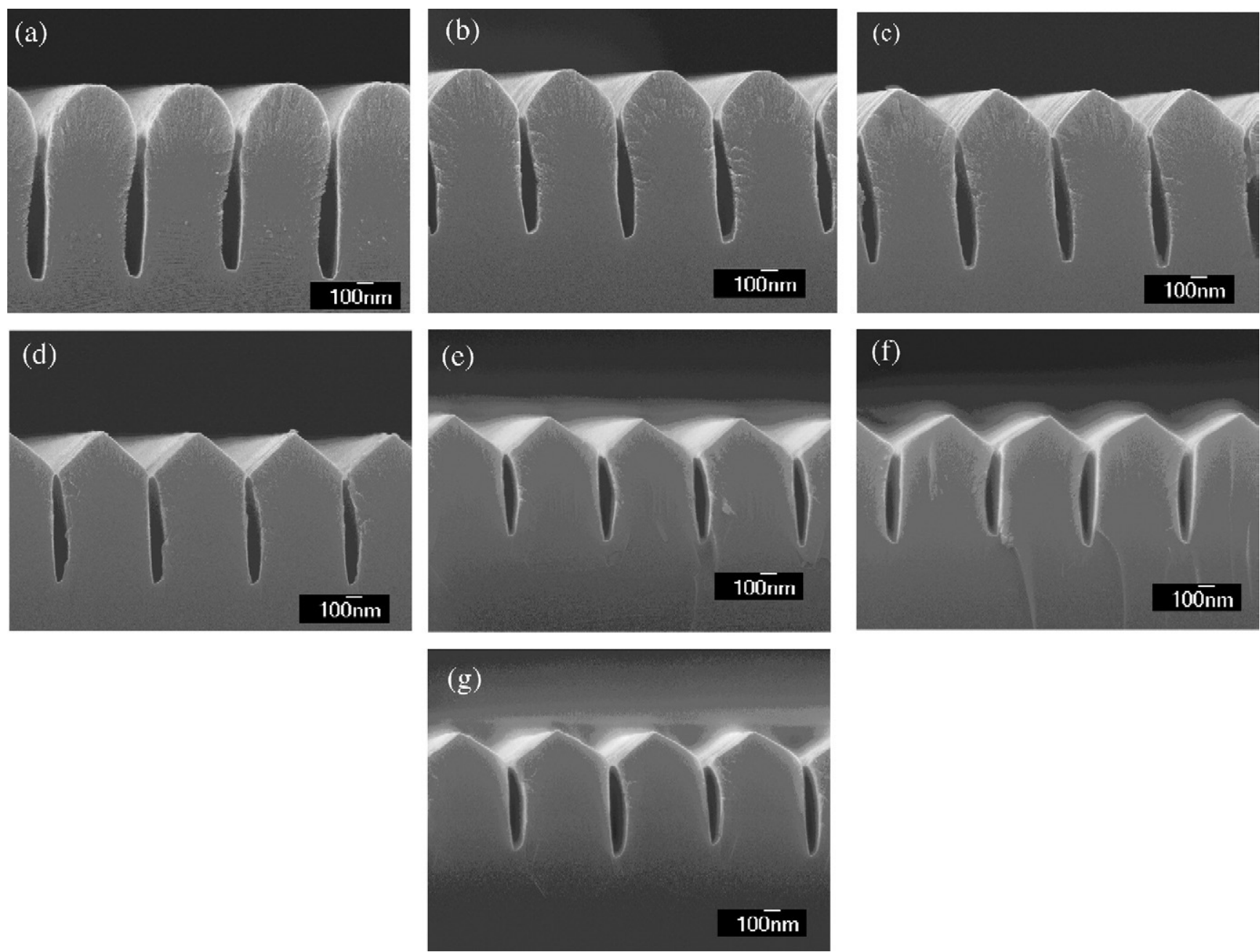

Fig. 5. IAD Autocloning process with ion-etching times of (a) $0 \mathrm{~min}$, (b) $20 \mathrm{~min}$, (c) $40 \mathrm{~min}$, (d) $60 \mathrm{~min}$, (e) $80 \mathrm{~min}$, (f) $100 \mathrm{~min}$ and (g) $120 \mathrm{~min}$.

show that the ridges of the adjusting layers eroded and the surface became flatter as the ion-beam voltages were increased. In short, $400 \mathrm{~V}$ was the best ion-beam voltage for our selfshaping autocloning fabrication process.

\subsection{Effect of the ion-etching time}

In the previous section it is shown that when the adjusting layers are formed under appropriate conditions the surface shape remains stationary. Actually, if after deposition the shape of the adjusting layer is not yet perfect triangular ion-beam etching can be used to improve the shape, making it closer to a perfect zigzag structure. Ion-beam etching can also be used to modify the slope of the corrugation by controlling the ionetching time.

In the previous section it became clear that an ion-beam voltage of $400 \mathrm{~V}$ was the best for forming a zigzag structure. So, we used the same ion-beam parameters for substrates with grooved structures with a period of $0.6 \mu \mathrm{m}$ and a depth of $0.3 \mu \mathrm{m}$. After thin-film deposition, additional ion-beam bombardment was applied to etch and adjust the surface shape, the so-called "self-shaping process". Fig. 5(a), (b), (c), (d),(e), (f) and (g) show the patterns after ion etching for $0 \mathrm{~min}$, $20 \mathrm{~min}, 40 \mathrm{~min}, 60 \mathrm{~min}, 80 \mathrm{~min}, 100 \mathrm{~min}$ and $120 \mathrm{~min}$, respectively.

Without ion etching, after the deposition process, as shown in Fig. 5(a), the shape of the adjusting layer did not form a perfect triangular shape. When the time of the ion etching increased to $40 \mathrm{~min}$, as shown in Fig. 5(c), the surface shape of the adjusting layer was modified to become a good zigzag

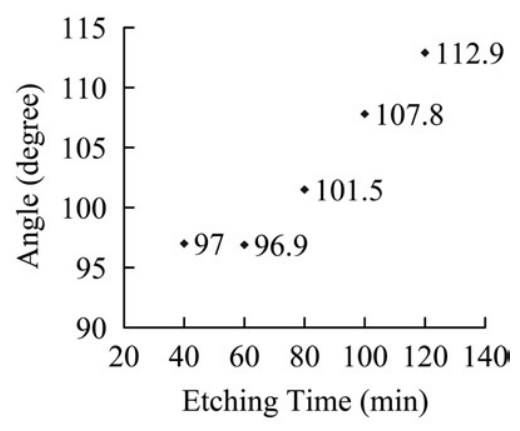

Fig. 6. Angle of the corrugation slope vs ion-etching time. 

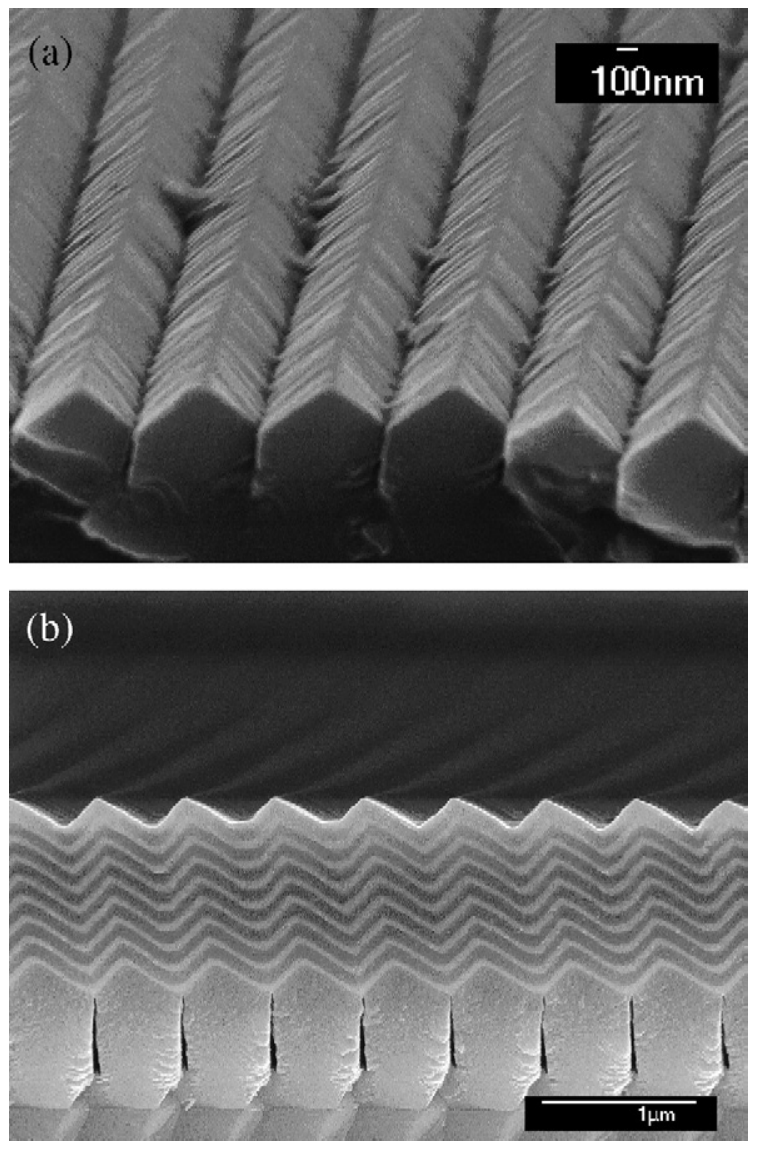

Fig. 7. Cross-sectional photographs of (a) the $\mathrm{SiO}_{2}$ adjusting layer (b) the stacked $\mathrm{Ta}_{2} \mathrm{O}_{5} / \mathrm{SiO}_{2}$ multilayers.

structure. As the ion-etching time was increased, the corrugation slope became flatter and flatter. Fig. 6 shows the relationship of the ion-etching time to the angle of the corrugation slopes. The results show that the angle increased as the ion-etching time increased. From these experimental data, it can be seen that the surface shape of the zigzag structure necessary to design $3 \mathrm{D} \mathrm{PhCs}$ can be controlled.

\section{Results and discussion}

We combined the lithographic and thin-film deposition IAD E-beam gun technologies to fabricate 3D PhCs. The lithographic technology provided the 2D periodic surface corrugation while the thin-film IAD E-beam gun deposition process preserved the periodic surface of the corrugation even after the deposition of multilayer stacks on the periodic corrugation patterns of the substrates, and also provided the third dimension of the periodic structure. The results show that the periodic surface corrugations were preserved very well after the deposition of multilayer structures under the appropriate IAD parameters. According to previous analyses, when films are formed under appropriate conditions, the surface shapes remain stationary. In addition the ion-etching time can be used to control the corrugation slopes of the adjusting layers.
Utilizing the above techniques, 3D PhCs can be fabricated by an E-beam gun with an IAD process. Periodic striped corrugation patterns were first patterned by electron-beam lithography on silicon wafers: the grooves had $0.6 \mu \mathrm{m}$ periods, $0.36 \mu \mathrm{m}$ widths, and $0.3 \mu \mathrm{m}$ depths. The $\mathrm{SiO}_{2}$ deposition thickness was about $0.8 \mu \mathrm{m}$. After deposition, the adjusting layer was bombarded by a $400 \mathrm{~V}$ ion-beam for $80 \mathrm{~min}$. Fig. 7(a) shows a cross-section of the adjusting layer. The angle of the corrugation slopes was about $102^{\circ}$.

The complete adjusting layer consisted of 17 alternate $\mathrm{Ta}_{2} \mathrm{O}_{5} / \mathrm{SiO}_{2}$ multilayers. The experimental parameters are shown in Table 1. Fig. 7(b) shows a cross-section of the stacked $\mathrm{Ta}_{2} \mathrm{O}_{5} / \mathrm{SiO}_{2}$ multilayers. The angle of the corrugation slopes was about $102^{\circ}$, about the same as that of the adjusting layer. The results show that the periodic surface corrugation was very well preserved even after the deposition of the multilayered structures.

\section{Conclusion}

In this study, 3D PCs were fabricated by using E-beam gun evaporation followed by ion-assisted deposition (IAD). We analyzed the effects of the different parameters, including the depths of the grooves, the ion-beam voltage and the ion-etching time in the autocloning process needed to form an adjusting layer with a good zigzag structure. We found that without changing the corrugation pattern of the substrate, the corrugation slopes could be adjusted between $96.9^{\circ}$ and $112.9^{\circ}$ simply by controlling the parameters of ion beam. Finally, the periodic surface corrugation pattern was preserved even after the deposition of the multilayer stacks to fabricate 3D PCs. The results show that the periodic surface corrugation performed very well after the deposition of multilayers with appropriate IAD parameters.

\section{Acknowledgements}

The authors would like to thank Delta Electronic Inc. and the National Science Council of Taiwan for the financial support of this research under Contract No. NSC94-2622-E-008-017.

\section{References}

[1] E. Yablonovitch, Phys. Rev. Lett. 58 (1987) 2059.

[2] S. John, Phys. Rev. Lett. 58 (1987) 2486.

[3] B. Gralak, G. Tayeb, S. Enoch, Opt. Express 9 (2001) 567.

[4] A.R. Parker, R.C. McPhedran, D.R. McKenzie, L.C. Botten, N.P. Nicorovici, Nature 409 (2001) 36.

[5] J. Zi, X. Yu, Y. Li, X. Hu, C. Xu, X. Wang, X. Liu, R. Fu, Proceedings of the National Academy of Sciences, USA, vol. 100, 2003, p. 12576.

[6] P. Vukusic, J.R. Sambles, Nature 424 (2003) 852.

[7] S. Kawakami, Electron. Lett. 13 (1997) 1260.

[8] S. Kawakami, T. Sato, K. Miura, Y. Ohtera, T. Kawashima, H. Ohkubo, IEEE Photonics Technol. Lett. 15 (2003) 816.

[9] H.L. Chen, H.F. Lee, W.C. Chao, C.I. Hsieh, F.H. Ko, T.C. Chu, J. Vac. Sci. Technol., B 22 (2004) 3359. 